\title{
Co-existence of VIM-2-producing Pseudomonas aeruginosa and KPC-2 and OXA-232-co-producing Klebsiella pneumoniae in the United States
}

\author{
Elizabeth Palavecino (D), M.D. ${ }^{1}$, Kacy Ramirez (D), M.D. ${ }^{2}$, Shermalyn R. Greene (D), Ph.D. ${ }^{3}$, and Abdullah Kilic (D, M.D. ${ }^{1}$ \\ ${ }^{1}$ Department of Pathology, Wake Forest School of Medicine, Winston-Salem, NC, USA; ${ }^{2}$ Department of Pediatrics, Wake Forest School of Medicine,
} Winston-Salem, NC, USA; ${ }^{3}$ NC State Laboratory of Public Health, Raleigh, NC, USA

\section{Dear Editor,}

Infections due to carbapenem-resistant gram-negative bacteria are a major health problem worldwide. Carbapenem resistance in gram-negative bacteria can result from a diversity of mechanisms: carbapenemase production, porin mutations, increased efflux pump activity, or the presence of enzymes other than carbapenemases. Most carbapenem-resistant isolates produce carbapenemase enzymes, which are found on plasmids that can be easily shared with other gram-negative bacteria [1]. The most common carbapenemase types in gram-negative bacteria are Klebsiella pneumoniae carbapenemase (KPC), New Delhi metallo- $\beta$-lactamase (NDM), Verona integron-encoded metallo$\beta$-lactamase (VIM), oxacillinase-48-type carbapenemases (OXA48), and imipenemase (IMP). A blaкpc-producing K. pneumoniae isolate was first recovered from a patient in North Carolina (NC), USA, in 1996 [2]. Since then, many reports of carbapenem-resistant Enterobacteriaceae (CRE) in hospitalized patients, particularly in the northeastern USA have been published. Carbapenem resistance is also an emerging problem among Pseudomonas aeruginosa isolates in many countries worldwide [3].

We report the co-existence of $\mathrm{VIM}$-2-producing $P$. aeruginosa and KPC-2 and OXA-232 co-producing K. pneumoniae isolates in a patient. To the best of our knowledge, this is the first report of two different species of carbapenemase-producing organisms isolated from the same patient sample in the USA. In addition, while some cases of OXA-232 and NDM co-production have been identified in the USA and subsequently around the world [4], to the best of our knowledge, KPC and OXA-232 (a variant of OXA-48) co-production in a single $K$. pneumoniae isolate has not been reported worldwide to date.

A pediatric patient less than five years old who had received care in India was transferred to a pediatric outpatient clinic at Wake Forest Baptist Health, Winston Salem, NC, in May 2019 with a two-year history of bilateral mucopurulent and malodorous ear discharge. The Institutional Review Board of Wake Forest School of Medicine approved the study for saving the isolate and performing additional testing (IRB approval no. IRB00008691). No informed consent was obtained since CRE is a reportable disease in NC.

The patient was diagnosed as having chronic otorrhea through perforated tympanic membranes, and the ear discharge fluid was sent to the clinical microbiology laboratory for bacterial culture. Both K. pneumoniae and P. aeruginosa were recovered from the ear sample, and the organisms were identified to the
Received: July 22, 2019

Revision received: September 5, 2019

Accepted: November 14, 2019

Corresponding author: Elizabeth Palavecino, M.D.

Department of Pathology, Wake Forest Baptist Medical Center, Medical Center Boulevard, Winston-Salem, NC 27157, USA

Tel: +1-336-716-2638, Fax: +1-336-716-7595

E-mail: epalave@wakehealth.edu

\section{(c) (1) $(9$}

(C) Korean Society for Laboratory Medicine

This is an Open Access article distributed under the terms of the Creative Commons Attribution Non-Commercial License (http://creativecommons.org/licenses/by-nc/4.0) which permits unrestricted non-commercial use, distribution, and reproduction in any medium, provided the original work is properly cited. 
species level using a matrix-assisted laser desorption/ionizationtime of flight system (Bruker Daltonics, Billerica, MA, USA). Antibiotic susceptibility testing was performed with the MicroScan WalkAway system (Beckman Coulter, West Sacramento, CA, USA) using Neg MIC Panel Type 45, and the E-test system (BioMérieux, Durham, NC, USA) was used for ceftazidime/avibactam and ceftolozane/tazobactam testing. The antibiotic susceptibility results are shown in Table 1.

Owing to carbapenem resistance, the isolates were evaluated for carbapenemase production using the modified carbapenem inactivation method; both isolates yielded a positive result indicating the presence of a carbapenemase. The presence of specific carbapenemase genes was determined using the Xpert

Table 1. Antibiotic susceptibility testing results for the K. pneumoniae and $P$. aeruginosa isolates

\begin{tabular}{|c|c|c|}
\hline \multirow{2}{*}{ Antibiotic(s) } & \multicolumn{2}{|c|}{ MIC $(\mu \mathrm{g} / \mathrm{mL})^{*}$} \\
\hline & K. pneumoniae & $P$. aeruginosa \\
\hline Ampicillin/sulbactam & $>16 / 8, R$ & NA \\
\hline Amikacin & $>32, \mathrm{R}$ & $>32, \mathrm{R}$ \\
\hline Amoxicillin/clavulanate & $>16 / 8, R$ & NA \\
\hline Aztreonam & $>16, \mathrm{R}$ & $8, S$ \\
\hline Ceftriaxone & $>32, \mathrm{R}$ & NA \\
\hline Ceftazidime & $>16, \mathrm{R}$ & 16,1 \\
\hline Cefoxitin & $>16, \mathrm{R}$ & NA \\
\hline Cefazolin & $>16, R$ & NA \\
\hline Ciprofloxacin & $>2, \mathrm{R}$ & $>2, \mathrm{R}$ \\
\hline Cefepime & $>16, R$ & 16,1 \\
\hline Ertapenem & $>1, \mathrm{R}$ & NA \\
\hline Gentamicin & $>8, \mathrm{R}$ & $>8, \mathrm{R}$ \\
\hline Imipenem & $4, \mathrm{R}$ & $>8, R$ \\
\hline Meropenem & $8, \mathrm{R}$ & $>8, R$ \\
\hline Piperacillin/tazobactam & $>64, \mathrm{R}$ & $16, S$ \\
\hline Trimethoprim-sulfamethoxazole & $\leq 2 / 38, S$ & NA \\
\hline Tetracycline & $\leq 4, S$ & NA \\
\hline Tigecycline & $\leq 2, S$ & NA \\
\hline Ticarcillin/clavulanate & $>64, \mathrm{R}$ & $>64, \mathrm{R}$ \\
\hline Tobramycin & $>8, R$ & $>8, R$ \\
\hline Ceftolozane/tazobactam & $32 / 4, \mathrm{R}$ & $128 / 4, \mathrm{R}$ \\
\hline Ceftazidime/avibactam & $1 / 4, S$ & $16 / 4, \mathrm{R}$ \\
\hline
\end{tabular}

*MICs were determined using the MicroScan WalkAway system (Beckman Coulter West Sacramento, CA, USA) with the Neg MIC Panel Type 45, except for ceftolozane/tazobactam and ceftazidime/avibactam, which were determined using the E-test.

Abbreviations: MIC, minimum inhibitory concentration; NA, not applicable; $\mathrm{R}$, resistant; I, intermediate; S, susceptible (based on the CLSI guidelines [10]).
Carba-R test (Cepheid, Sunnyvale, CA, USA). The blakpc and blaoxA-48 genes were detected in the K. pneumoniae isolate, and the blavim gene was detected in the $P$. aeruginosa isolate. The Xpert Carba- $R$ test does not provide differentiation of the variants, and therefore further characterization was performed. Both isolates were sent to the North Carolina State Laboratory of Public Health (NCSLPH), where the original findings were confirmed. Next generation sequencing and subsequent genomic analysis were performed as previously described [5]. Sequencing data demonstrated that the $K$. pneumoniae isolate had the blaкрс-2, blaохA-232, and blactx-M-15 genes, and the $P$. aeruginosa isolate had the blavim-2 gene.

The patient was treated with topical ciprofloxacin/dexamethasone and oral trimethoprim-sulfamethoxazole for 10 days; subsequently, the patient returned to the pediatric outpatient clinic 19 days later for follow-up. His ear discharge had partially improved. A new ear sample collected during the second visit yielded VIM-2 producing $P$. aeruginosa, but not $K$. pneumoniae. He was seen by a pediatric otolaryngologist and ultimately clinically responded to aural debridement and 21 days of topical acetic acid $2 \%$ solution.

KPC-producing CRE are the most common carbapenemaseproducing CRE (CP-CRE) in the USA [1]. CP-CRE strains other than $\mathrm{KPC}$ remain extremely rare as a cause of infections in the USA and are often related with health care exposure outside the USA. In 2013, the first description of two OXA-48-like-producing $\mathrm{CRE}$ isolates collected during a surveillance study was published in the USA [6]. OXA carbapenemases have been divided into 14 variants based on amino acid sequences; OXA-232 is a five amino acid variant of OXA-48 first identified in France in 2011 [7]. In 1999, VIM family class B metallo- $\beta$-lactamases were initially described in Acinetobacter spp. and $P$. aeruginosa isolates in Europe. The VIM family has been subsequently detected in Enterobacteriaceae isolates such as Escherichia coli and K. pneumonia. In the USA, a $P$. aeruginosa isolate carrying blavim, a mobile metallo- $\beta$-lactamase gene was first recovered in 2001 from a 58-year-old female with a history of autoimmune hepatitis that required a liver transplant [3]. Following this case, a nosocomial outbreak due to pan-resistant VIM-producing $P$. aeruginosa isolates was reported in a public teaching hospital in Chicago [8].

Co-producing isolates are a public health risk as they tend to have higher carbapenem minimum inhibitory concentrations than strains harboring individual carbapenemase genes. These isolates can be a source of mobile genetic elements carrying resistance genes and are also easily able to transfer antibiotic resistance to other organisms via mobile elements [9]. 
This report highlights the importance of hospital staff to be aware of organisms that may produce carbapenemases and of investigating whether a patient has received health care in countries known to have high rates of carbapenemase-producing organisms. Clinical laboratories should have the capacity to provide early detection of carbapenemase-producing bacteria to prevent the spread of these resistant organisms in both community and hospital settings.

\section{ACKNOWLEDGEMENTS}

The authors thank the CDC, the NCSLPH Molecular Diagnostics and Molecular Epidemiology Unit, and the Maryland Public Health Laboratories for providing the sequencing information of the carbapenemases. The authors also thank Carlos A. Fasola for helpful editorial suggestions.

\section{AUTHOR CONTRIBUTIONS}

Elizabeth Palavecino supervised the testing, reviewed the data and provided the outline for the manuscript, reviewed and edited the manuscript revised versions and final version.

Shermalyn Greene provided the additional molecular data and the NC Health department epidemiologic information.

Kacy Ramirez provided the clinical information and follow up of the case.

Abdullah Kilic prepared the draft and reviewed the references.

\section{CONFLICTS OF INTEREST}

None declared.

\section{RESEARCH FUNDING}

The work performed at the NCSLPH was supported by funding from the Epidemiology and Laboratory Capacity for Prevention and Control of Emerging Infectious Diseases (ELC) Grant.

\section{ORCID}

Elizabeth Palavecino https://orcid.org/0000-0002-4525-6064

Kacy Ramirez

Shermalyn R. Greene https://orcid.org/0000-0002-1773-8568 https://orcid.org/0000-0001-9975-5398

Abdullah Kilic https://orcid.org/0000-0003-0875-6593

\section{REFERENCES}

1. Livorsi DJ, Chorazy ML, Schweizer ML, Balkenende EC, Blevins AE, Nair $\mathrm{R}$, et al. A systematic review of the epidemiology of carbapenem-resistant Enterobacteriaceae in the United States. Antimicrob Resist Infect Control 2018; 7:55.

2. Yigit H, Queenan AM, Anderson GJ, Domenech-Sanchez A, Biddle JW, Steward CD, et al. Novel carbapenem-hydrolyzing beta-lactamase, KPC1 , from a carbapenem-resistant strain of Klebsiella pneumoniae. Antimicrob Agents Chemother 2001;45:1151-61.

3. Toleman MA, Rolston K, Jones RN, Walsh TR. blavim-7, an evolutionarily distinct metallo-beta-lactamase gene in a Pseudomonas aeruginosa isolate from the United States. Antimicrob Agents Chemother 2004;48:32932.

4. Doi Y, O'Hara JA, Lando JF, Querry AM, Townsend BM, Pasculle AW, et al. Co-production of NDM-1 and OXA-232 by Klebsiella pneumoniae. Emerg Infect Dis 2014;20:163-5.

5. Senchyna F, Gaur RL, Sandlund J, Truong C, Tremintin G, Kültz D, et al. Diversity of resistance mechanisms in carbapenem-resistant Enterobacteriaceae at a health care system in Northern California, from 2013 to 2016. Diagn Microbiol Infect Dis 2019:250-7.

6. Lascols C, Peirano G, Hackel M, Laupland KB, Pitout JD. Surveillance and molecular epidemiology of Klebsiella pneumoniae isolates that produce carbapenemases: first report of OXA-48-like enzymes in North America. Antimicrob Agents Chemother 2013;57:130-6.

7. Potron A, Rondinaud E, Poirel L, Belmonte O, Boyer S, Camiade S, et al. Genetic and biochemical characterisation of OXA-232, a carbapenem-hydrolysing class D $\beta$-lactamase from Enterobacteriaceae. Int J Antimicrob Agents 2013;41:325-9.

8. Lolans K, Queenan AM, Bush K, Sahud A, Quinn JP. First nosocomia outbreak of Pseudomonas aeruginosa producing an integron-borne metallo-beta-lactamase (VIM-2) in the United States. Antimicrob Agents Chemother 2005;49:3538-40.

9. Wang Y, Cao W, Zhu X, Chen Z, Li L, Zhang B, et al. Characterization of a novel Klebsiella pneumoniae sequence type 476 carrying both blakpc-2 and blaimp-4. Eur J Clin Microbiol Infect Dis 2012;31:1867-72.

10. CLSI. Performance standards for antimicrobial susceptibility testing, 29th ed. CLSI supplement M100-S29. Wayne, PA: Clinical and Laboratory Standards Institute. 2019. 